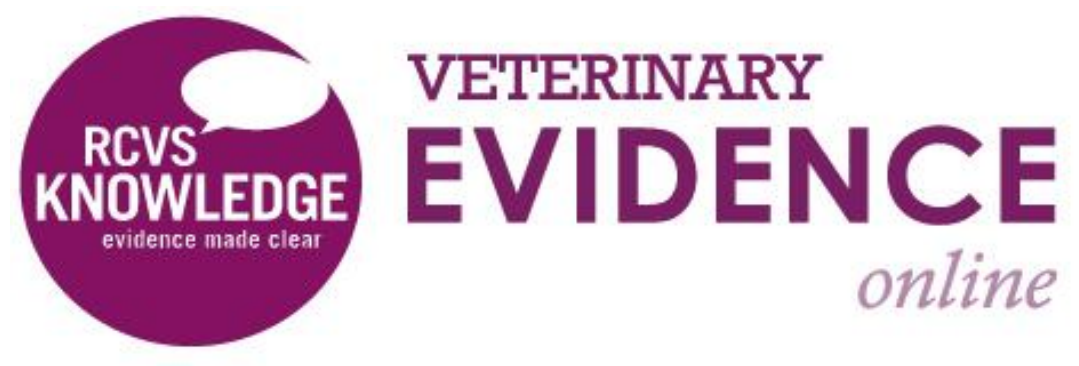

\title{
Supraspinatus Tendinopathy in 327 Dogs: A Retrospective Study
}

Sherman Orye Canapp, Jr. DVM, MS, CCRT, DACVS, DACVSMR ${ }^{{ }^{*}}$

Debra Ann Canapp DVM, CVA, CCRT, DACVSMR ${ }^{1}$

Brittany Jean Carr DVM, CCRT ${ }^{1}$

Catherine Cox MS ${ }^{1}$

Jennifer Genevieve Barrett PhD, DVM, DACVS, DACVSMR ${ }^{2}$

\footnotetext{
${ }^{1}$ Veterinary Orthopaedic and Sports Medicine Group, 10975 Guilford Road, Annapolis Junction, MD 20701

2 Marion duPont Scott Equine Medical Center, Virginia-Maryland College of Veterinary Medicine, 17690 Old Waterford Road Leesburg, VA 20176

Corresponding Author (scanapp@vosm.com)
}

ISSN: 2396-9776

Published: 04 July 2016

in: Vol 1, Issue 3

DOI: http://dx.doi.org/10.18849/ve.v1i3.32

Reviewed by: Wanda Gordon-Evans (DVM, PhD, DACVS DACVSMR) 
Objective: To report clinical findings and treatments for dogs with supraspinatus tendinopathy (ST).

Background: ST is a term used to describe tears, calcifying tendinopathy, tendinosis and/or injuries in and around the tendon of the supraspinatus muscle, and is a cause of forelimb lameness, especially in sporting and performance dogs.

Evidentiary value: This is a retrospective study of 327 dogs diagnosed with ST.

Methods: Medical records (2006 to 2013) were reviewed for history, signalment, prior treatments, physical examination findings, diagnostic imaging and arthroscopic findings, concurrent shoulder and elbow pathologies, and treatments performed.

Results: Dogs aged 4 months to 14 years (average 6.5 years; median 6 years) were diagnosed with ST. Performance and sporting dogs were $39.4 \%$ of the population, with $58.1 \%$ of them being agility dogs. Pain was elicited on palpation of the supraspinatus tendon in $49.3 \%$ of dogs. Shoulder radiographs in 283 dogs showed mineralisation in $13 \%$ of cases. MRI of the shoulder was performed in 31 cases and revealed findings indicative of ST, including hyperintensity of signal on T1 weighted image (or "spin-lattice") and Short T1 Inversion Recovery (STIR) sequences of the supraspinatus tendon at its insertion on the greater tubercle and mineralisation of the supraspinatus tendon. Common ultrasonographic findings included increased tendon size (76\%), irregular fibre pattern (74\%), and non-homogeneous echogenicity (92.5\%). The most common findings on shoulder arthroscopy were supraspinatus bulge (82.2\%) and subscapularis pathology (62.4\%). Elbow pathology was recorded in $54.5 \%$ of dogs. Treatment outcomes showed $74.6 \%$ of dogs failed to respond to non-steroidal anti-inflammatory drugs (NSAID) and $40.8 \%$ failed to respond to rehabilitation.

Conclusions: These findings suggest concurrent shoulder and/or elbow pathology is not uncommon in dogs with ST. Further, ST often fails to respond to NSAID therapy and rehabilitation therapy.

Application: Other treatments such as regenerative medicine should be considered. Future studies are still needed to assess the use and efficacy of regenerative medicine for ST.

INTRODUCTION

The shoulder joint of the dog is distinct in its ability to withstand large forces and allow for great mobility while maintaining the control needed for stable and precise function of the forelimb. Both the stability and function of the shoulder joint relies on a complex network of supportive soft tissue structures, specifically those of the rotator cuff which include the supraspinatus, infraspinatus, teres minor, and subscapularis (1-4). The supraspinatus is an important passive stabiliser of the shoulder joint, and is responsible for shoulder extension and advancing the limb $(3,4)$. Several degenerative disorders have been identified in both humans and dogs involving the supraspinatus tendon including rotator cuff tears, calcifying tendinopathy (bony mineralisation within the tendon) and tendinosis (microtears within the tendon) (3,5-7). ST is a term used to describe these clinical conditions and/or injuries in and around the tendon of the supraspinatus muscle, and is a cause of forelimb lameness, especially in human athletes and performance dogs $(5,8-14)$.

The aetiology of ST in performance dogs is thought to be repeated strain activity and overuse from chronic repetitive activity, with a failure of adequate remodeling $(8,10,13,14)$. While inflammation may play a role in the initiation of ST, it generally does not in the propagation and progression of the disease process. Histology of pathologic supraspinatus tendons shows either absent to minimal inflammation with hypocellularity, a loss 
of tightly bundled collagen appearance, an increased proteoglycan content, and lack of neovascularisation in response to injury (5,14-22). Tendons damaged from repeated strain demonstrate discontinuous, disorganised tendon fibres with little to no inflammation and occasional mineralisation within the tendon and bony remodeling at its insertion site in chronic cases $(14,21)$. In chronic cases, calcification at the site of insertion has been well documented in both humans and dogs $(17,22-25)$.

Diagnosis of ST can be made on the basis of a combination of orthopaedic examination, imaging, and diagnostic arthroscopic findings. On initial presentation dogs with ST typically present with a weight bearing lameness on the affected limb that often worsens with activity. Direct palpation of the tendon and flexion of the shoulder commonly produces pain, and the supraspinatus muscle may be atrophied $(10,22,25,26-28)$. Mineralisation of the supraspinatus tendon can be identified with diagnostic imaging modalities such as radiography, computerised tomography, magnetic resonance imaging (MRI), and diagnostic ultrasonography $(10,17,24,29-31)$. The most common finding on MRI is enlargement and increased signal in the area of insertion of the supraspinatus tendon over the greater tubercle of the humerus on T2 Weighted Image (T2-W) and STIR sequences $(10,32)$. Diagnostic musculoskeletal ultrasonography is a relatively new technique that has been used and validated for the diagnosis of ST in both humans and dogs (8,33-40). Musculoskeletal ultrasound provides a non-invasive definitive diagnosis of ST, and allows for facile and cost-effective sequential exams to assess response to treatment $(8,33-40)$. Changes in size, shape, and echogenicity of the tendon found on diagnostic ultrasounds indicate ST.

Conservative treatment of ST includes medical management of acute inflammation, controlled activity, rehabilitation therapy, acupuncture, deep cross-friction massage, heat, ultrasound therapy, range of motion (ROM) and stretching, and therapeutic laser therapy. Extracorporeal shockwave therapy has also been used in chronic calcified ST in both humans and dogs $(2,14,42-44)$. As in humans, other treatment options include regenerative medicine therapy and surgery $(10,14,16,17)$. Platelet-rich plasma (PRP), stem cell therapy (SCT), or a combination of these may be directly injected into the tendon under ultrasonographic guidance. Surgical intervention (debulking or releasing the supraspinatus tendon) has been documented in chronic cases that do not respond to conservative management and rehabilitation therapy. In addition, arthroscopic evaluation of the shoulder can be performed to rule out concurrent pathologies, such as medial shoulder syndrome. Rehabilitation therapy as previously described is crucial following any surgical treatment (45). Healing can be confirmed by improvement in lameness, pain on palpation, improved range of motion, and improvement in appearance of the tendon using MRI and/or musculoskeletal ultrasonography.

While there is a great deal of literature regarding the presentation, clinical findings, diagnostic imaging characteristics of ST in humans, to the authors' knowledge there is limited literature describing ST in dogs. The purpose of this retrospective study is to describe clinical findings and diagnostic imaging characteristics for dogs with ST.

\section{METHODS \& MATERIALS}

Medical records of client-owned dogs diagnosed with ST were reviewed from 2006-2013. Data collected included signalment, history, limb involvement, prior treatments, physical and orthopaedic examination, diagnostic imaging results (radiography, MRI, diagnostic musculoskeletal ultrasound), arthroscopy findings, presence of concurrent shoulder and elbow pathologies, and treatments performed. Dogs were included if a diagnosis of ST was made based on a combination of orthopaedic examination findings and diagnostic imaging findings. Dogs were excluded if medical records were incomplete. 
Dogs:

A total of 327 dogs were diagnosed with ST from 2006-2013. All records were found to be complete. Ages ranged from 4 months to 14 years (average 6.5 years; median 6 years). No sex predisposition was apparent, as there were 142 female dogs ( 20 intact) and 185 male dogs (41 intact). The different breed representations are displayed in Table 1. Performance and sporting dogs accounted for $39.4 \%$ of the population, and companion animals accounted for the remaining $60.6 \%$. Of the performance and sporting dog population, $58.1 \%$ participate in agility. At presentation, a majority (74.6\%) of dogs failed to respond to NSAID therapy, and $40.8 \%$ failed to respond to a dedicated rehabilitation therapy program for ST, which consisted of weekly rehabilitation therapy sessions for a minimum of 8 weeks.

Unilateral lameness was more common than bilateral, $62.4 \%$ and $37.6 \%$ respectively. The average lameness score, graded from 0 (sound) to 6 (non-weight bearing lame), was 2.1 (median 3). Duration of lameness at initial presentation ranged from one week to over one year. Less than one third of dogs (26.6\%) had a chronic (>1 year) lameness. Of the remaining, average duration was 14.7 weeks (median 13 weeks).

\section{Physical Examination Findings:}

A physical examination was performed on all 327 dogs. On physical examination, pain on direct palpation of the supraspinatus was found in $49.3 \%$ of dogs. Pain/spasm on shoulder extension and abduction was noted in $59.5 \%$ and $59.9 \%$, respectively. Pain/spasm on shoulder flexion was recorded to be found in $64.1 \%$, and pain on biceps stretch was recorded in $48 \%$ of dogs.

\section{Radiography:}

Shoulder radiographs were performed in 283 dogs (86.5\%). Mineralisation was noted in 37 cases (13\%) (Figure 1). In the 123 dogs with bilateral ST, mineralisation was noted in 10 cases; 8 unilateral and 2 bilateral. Elbow radiographs were performed in 267 dogs ( 480 elbows), and 76 of those dogs ( $28.3 \%$ of total ST dogs) had signs of elbow disease. Sclerosis was noted in $28.3 \%$ of elbows and degenerative joint disease (DJD) in $15.9 \%$.

\section{Magnetic Resonance Imaging:}

MRI of the shoulder was performed on 31 dogs. All MRI images were reviewed by an American College of Veterinary Radiology certified veterinary radiologist. Of these $31 \mathrm{dogs}, 50 \%$ of dogs had findings indicative of ST including hyperintensity of signal on T1 and STIR sequences of the ST at its insertion on the greater tubercle (Figure 2). A flattened or oval appearance of the biceps tendon, loss of fluid around the biceps tendon within the bicipital groove at the level of insertion of the supraspinatus, and/or displacement of the biceps tendon from the bicipital groove characterised biceps impingement secondary to ST in 50\% of cases (Figures 3 and 4). Fatty replacement at the myotendinous junction (seen on the T1 weighted image) of the supraspinatus was present in $30 \%$ of cases. Mineralisation of the ST was noted in $16 \%$ of cases.

\section{Diagnostic Musculoskeletal Ultrasonography:}

Diagnostic musculoskeletal ultrasonography was performed in 255 dogs (78\%). The supraspinatus tendon was evaluated in both the longitudinal and sagittal plane for each of the following characteristics: size, shape, echogenicity, and boney irregularity at the insertion. The following changes were also reported if present: biceps tendon pathology, synovial proliferation, and increases in synovial fluid volume within the biceps sheath and/or shoulder joint. Of the 255 cases, mixed echogenicity was found in $89.2 \%$ of cases, irregular fibre pattern was found in $52.5 \%$ of cases, the supraspinatus appeared enlarged in $50.6 \%$ of cases, a core lesion was 
identified in $23.6 \%$ of cases, boney irregularity was found in $19.3 \%$ of cases, bicipital tenosynovitis was found in $15.7 \%$ of cases, and mineralisation of the supraspinatus tendon was appreciated in $15 \%$ of cases (Figures 5 and 6).

\section{Arthroscopy:}

Shoulder arthroscopy was performed in 218 dogs. Of these, elbow arthroscopy was performed in 168 dogs; 23 dogs had elbow arthroscopy only. A total of 271 shoulders were evaluated via arthroscopy. Of the 271 shoulders, a supraspinatus bulge was noted in $82.2 \%$ of dogs (Figure 7). Of these, biceps tendon impingement was noted in $38.7 \%$ of dogs. While $29.2 \%$ of dogs had bicipital tenosynovitis, biceps pathology was noted in $37.6 \%$. Pathology of the subscapularis was observed in $62.4 \%$ of dogs; medial glenohumeral ligament (MGL) pathology was present in $44.6 \%$; labral tear in $32.8 \%$; cartilage defects in $21.8 \%$; and bone eburnation or osteophytosis in $11.1 \%$ of dogs. Of the 168 dogs evaluated via elbow arthroscopy, 257 elbows in total were evaluated. Of the 257 elbows evaluated, $54.5 \%$ had elbow pathology, of which $34.6 \%$ had fragmented medial coronoid processes, $5.1 \%$ had evidence of medial coronoid disease, and $14.8 \%$ had end stage medial compartment disease. The remaining $45.5 \%$ were within normal limits.

DISCUSSION

A total of 327 dogs were diagnosed with ST from 2006-2013 representing the largest accumulation of supraspinatus tendon injuries in a retrospective study to date. There was no apparent age, sex, or breed predisposition. Diagnostic musculoskeletal ultrasonography of the shoulder was performed in 255 of the 327 dogs. Changes consistent with ST were noted on diagnostic musculoskeletal ultrasound in the majority of cases. While the diagnostic musculoskeletal ultrasonographic grading scores and their significance are still being assessed, diagnostic musculoskeletal ultrasonography should be considered as a useful imaging modality in diagnosing ST. Musculoskeletal ultrasonography has several advantages over MRI. It is quicker, more economical, and can be performed without anesthesia or sedation in most instances. Also, sequential ultrasonographic examinations during the course of treatment allows for monitoring of treatment response. Recently, the Ultrasound Shoulder Pathology Rating Scale (USPRS) has been developed in humans (41). This scale provides an objective quantification of the pathology observed and is used not only for diagnosis but also monitoring treatment and progression of disease. Further study is needed to establish an USPRS for dogs.

Diagnostic arthroscopy was useful in the identification of ST, which has not been described in the primary literature. A supraspinatus bulge was noted in $82.2 \%$ of the 218 dogs that had shoulder arthroscopy performed highlighting the importance of a thorough evaluation of the shoulder during arthroscopic exploration.

Additionally, concurrent shoulder and/or elbow pathology was a common finding on arthroscopy. Pathology consistent with medial shoulder syndrome was found in $62.4 \%$ of dogs. Of the 257 elbows in 191 dogs that were evaluated via arthroscopy, elbow pathology (fragmented medial coronoid process and/or medial compartment disease) was found in $54.5 \%$ of dogs. These findings suggest that concurrent shoulder and/or elbow pathology is not uncommon in dogs with ST. Our finding that concurrent injury to other forelimb structures is common also suggests the same repetitive actions that cause ST can also cause other forelimb injuries, such as traumatic fragmentation of the medial coronoid process (TFMCP), thereby increasing lameness of the affected limb $(4,15)$. However, it is also possible that pathologies such as non-traumatic fragmented medial coronoid process (FMCP) or medial compartment disease (MCD) may not only exist concurrently but also potentially predispose patients to compensatory shoulder pathology, such as ST $(4,15)$. This suggests that ST may be secondary in these cases and that treating the primary pathology should be considered when formulating a treatment plan.

When considering treatment options for ST dogs, it is important to note that many of the patients failed to respond to conservative management prior to presentation as $74.6 \%$ of patients failed to respond to NSAID 
therapy and $40.8 \%$ failed to respond to rehabilitation therapy. This may suggest that conservative management is often insufficient to treat ST. While dogs treated surgically have been shown to have improvements in gait and overall comfort, regenerative medicine therapy also shows promising results and should be considered for dogs with ST $(2,10,27)$.

Treatment of acute cases of ST consists of conservative management including medical management, rehabilitation therapy, controlled activity, and acupuncture; however, treatment of chronic cases of ST is somewhat different. Dogs tend to take longer to recover due to the chronic nature of the changes in the tendon, as well as take longer to heal and progress through the different stages of exercises. The inflammatory process must be reinitiated in order to break down scar tissue and remodel the fibres of the tendon into their proper orientation, thus increasing its load capacity. NSAIDs are not recommended for chronic cases of ST because of the need to reinitiate the inflammatory response $(2,4)$. Treatment of chronic cases of ST often includes deep cross-friction massage, heat, ultrasound therapy, ROM and stretching. Acupuncture and cold laser therapy can also be utilised to increase circulation and reduce pain. Extracorporeal shockwave therapy has also been used in chronic calcified ST in both humans and dogs $(2,14,42-44)$.

Regenerative medicine therapy has also been utilised for ST in both humans and dogs, especially for cases where a core lesion is present $(1,7,14,43,44,48-51)$. Platelet-rich plasma (PRP), stem cell therapy (SCT), or a combination of these may be directly injected into the tendon under ultrasound guidance. Severe cases may require a second injection $(1,44,49,50)$. This therapy may be used as a primary treatment, however; arthroscopic exploration of the shoulder is recommended in conjunction since concurrent pathologies are not uncommon. Arthroscopic treatments may be combined with an intra-articular injection of regenerative medicine therapy to aid in and speed the healing and recovery process (2). Future studies are needed to evaluate the use of regenerative medicine therapy for ST and other tendon injuries in the canine.

The major limitation of this study is that it is a retrospective study. Shortcomings can include recall bias, missing data points and lost follow-up. For evaluation of treatments and outcomes, lack of randomisation and the influence of bias and confounders also come into play. However, the large number of cases identified in this study contributes a significant database to the current literature, until further controlled studies that objectively measure treatment outcomes are performed. Of note, the common clinical findings and diagnostic imaging characteristics of ST have been thoroughly described.

In conclusion, these findings suggest concurrent shoulder and/or elbow pathology is not uncommon in dogs with ST. Further, chronic ST often fails to respond to NSAID therapy and rehabilitation therapy, suggesting that other treatments such as regenerative medicine should be considered. Future studies are still needed to assess the use and efficacy of regenerative medicine for ST.

\section{CONFLICT OF INTEREST}

The authors declare no conflicts of interest.

\section{REFERENCES}

1. Marcellin-Little, D.J. Levine, D. and Canapp S.O. (2007) The Canine Shoulder: Selected Disorders and Their Management with Physical Therapy. Clinical Techniques in Small Animal Practice, 22 (4), pp. 171182. http://dx.doi.org/10.1053/j.ctsap.2007.09.006

2. Canapp, S.O. and Gross Saunders, D. (2014) Common Conditions and Physical Rehabilitation of the Athletic Patient. In: Millis, D.L, Levine, D, Canine Rehabilitation and Physical Therapy. Second ed. Philadelphia, Elsevier Saunders. 
3. Canapp, S.O. (2013) Supraspinatus Tendinopathy in Dogs. In: Proceedings ACVS Symposium. San Antonio, TX 24 -26 April. American College of Veterinary Sports Medicine and Rehabilitation, pp. $504-$ 506.

4. Canapp, S.O. and Kirkby, K.A. (2013) Disorders of the Thoracic Limb. In: Zink M.C, Van Dyke J.B (eds), 223-249.

5. Kujat, R. (1990) The Microangiographic Pattern of the Rotator Cuff of the Dog. Archives of Orthopaedic and Trauma Surgery, 109 (2), pp. 68-71. http://dx.doi.org/10.1007/BF00439381

6. Sano, H. et al. (1997) Degeneration at the Insertion Weakens the Tensile Strength of the Supra Spinatus Tendon: A Comparative Mechanical and Histological Study of the Bone-Tendon Complex. Journal of Orthopaedic Research, 15 (5), pp. 719-

726. http://dx.doi.org/10.1002/jor.1100150514

7. Fransson, B.A. Gavin, P.R. and Lahmers, K.K. (2005) Supraspinatus Tendinosis Associated with Biceps Brachii Tendon Displacement in a Dog. Journal of the American Veterinary Medical Association, 227 (9), pp. 1429-1433. http://dx.doi.org/10.2460/javma.2005.227.1429

8. Arend, C.F. Arend, A.A. and Silva, T.R. (2014) Diagnostic Value of Tendon Thickness and Structure in the Sonographic Diagnosis of Supraspinatus Tendinopathy: Room for a Two-Step Approach. European Journal of Radiology, 83 (6), pp. 975-979. http://dx.doi.org/10.1016/i.ejrad.2014.02.021

9. Gilliland, B.C. (1994) Relapsing Polychondritis and Miscellaneous Arthritides. In: K.G. Isselbacher, E. Braunwald, J.D. Wilson, J.B. Martin, A.S. Fauci, D.L. Kasper, (ed) Harrison's principles of internal medicine. New York, NY: McGraw Hill. pp. 1706-1708.

10. Lafuente, M.P. et al. (2009) Surgical Treatment of Mineralized and Nonmineralized Supraspinatus Tendinopathy in Twenty-Four Dogs. Veterinary Surgery, 38 (3), pp. 380-

387.http://dx.doi.org/10.1111/j.1532-950X.2009.00512.x

11. Evans, H.E. (1993) Miller's Anatomy of the Dog, ed 3. Philadelphia, Saunders. Pp. 225-231.

12. Cullen, K.L. et al. (2013) Internet-Based Survey of the Nature and Perceived Causes of Injury to Dogs Participating in Agility Training and Competition Events. Journal of the American Veterinary Medical Association, 243 (7), pp. 1010-1018. http://dx.doi.org/10.2460/javma.243.7.1010

13. Lewis. J.S. (2009) Rotator Cuff Tendinopathy. British Journal of Sports Medicine, 43 (4) pp. 236241. http://dx.doi.org/10.1136/bjsm.2008.052175

14. Rees, J.D. Maffulli, N. and Cook, J. (2009) Management of Tendinopathy. The American Journal of Sports Medicine, 37 (9) pp. 1855-1867. http://dx.doi.org/10.1177/0363546508324283

15. Arrington, E.D. and Miller, M.D. (1995) Skeletal Muscle Injuries. The Orthopaedic clinics of North America, 26 (3) pp. 411-422.

16. Almekinders, L.C. and Temple, J.D. (1998) Aetiology, Diagnosis, and Treatment of Tendonitis: An Analysis of the Literature. Medicine \& Science in Sports \& Exercise, 30 (8) pp. 11831190.http://dx.doi.org/10.1097/00005768-199808000-00001

17. Hurt, G. and Baker, C.L. (2003) Calcific Tendinitis of the Shoulder. Orthopaedic Clinics of North America, 34 (4) pp. 567-575. http://dx.doi.org/10.1016/S0030-5898(03)00089-0

18. Hashimoto, T. Nobuhara, K. and Hamada, T. (2003) Pathologic Evidence of Degeneration as a Primary Cause of Rotator Cuff Tear. Clinical Orthopaedics and Related Research, 415 pp. 111120.http://dx.doi.org/10.1097/01.blo.0000092974.12414.22

19. Dean, B.J.F. Franklin, S.L. and Carr, A.J. (2012) A Systematic Review of the Histological and Molecular Changes in Rotator Cuff Disease. Bone \& Joint Research, 1 (7), pp. 158-166. 
20. Garcia, G.M. McCord G.C. and Kumar, R. (2003) Hydroxyapatite Crystal Deposition Disease. Seminars in Musculoskeletal Radiology, 7 (3), pp. 187-193. http://dx.doi.org/10.1055/s-2003-43229

21. Soslowsky, L.J. et al. (2000) Overuse Activity Injuries the Supraspinatus Tendon in an Animal Model: A Histologic and Biomechanical Study. Journal of Shoulder and Elbow Surgery, 9 (2), pp. 79-

84.http://dx.doi.org/10.1016/S1058-2746(00)90033-8

22. Fransson, B.A. Gavin, P.R. and Lahmers, K.K. (2005) Supraspinatus Tendinosis Associated with Biceps Brachii Tendon Displacement in a Dog. Journal of the American Veterinary Medical Association, 227 (9), pp. 1429-1433. http://dx.doi.org/10.2460/javma.2005.227.1429

23. Chung, C.B. Gentili, A. and Chew, F.S. (2004) Calcific Tendinosis and Periarthritis: Classic Magnetic Resonance Imaging Appearance and Associated Findings. Journal of Computer Assisted Tomography 28 (3), pp. 390-396. http://dx.doi.org/10.1097/00004728-200405000-00015

24. Rupp, S. Seil, R. and Kohn, D. (2000) Tendinosis Calcarea of the Rotator Cuff. Orthopade, 29 (10), pp. 852-67.

25. Kriegleder, H. (1995) Mineralization of the Supraspinatus Tendon: Clinical Observations in Seven Dogs. Veterinary and comparative orthopaedics and traumatology, 8, pp. 91-97.

26. Muir, P. et al. (1996) Force-Plate Analysis of Gait before and after Surgical Excision of Calcified Lesion of the Supraspinatus Tendon in Two Dogs. Veterinary Record, 139 (6), pp. 137-

139.http://dx.doi.org/10.1136/vr.139.6.137

27. Muir, P. and Johnson, K.A. (1994) Supraspinatus and Biceps Brachii Tendinopathy in Dogs. Journal of Small Animal Practice. 35 (5), pp. 239-243. http://dx.doi.org/10.1111/j.1748-5827.1994.tb03268.x

28. Kunkel, K.A. and Rochat, M.C. (2008) A Review of Lameness Attributable to the Shoulder in the Dog: Part Two. Journal of the American Animal Hospital Association, 44 (4) pp. 163-

170.http://dx.doi.org/10.5326/0440163

29. Flo, G.L. and Middleton, D. (1990) Mineralization of the Supraspinatus Tendon in Dogs. Journal of the American Veterinary Medical Association, 197 (1) pp. 95-97.

30. Laitinen, O.M. and Flor, G.L. (2000) Mineralization of the Supraspinatus Tendon in Dogs: A Long-Term Follow-Up. J Am Anim Hosp Assoc, 36 (3), pp. 262-267. http://dx.doi.org/10.5326/15473317-36-3-262

31. Friedman, L. et al. (2002) Ultrasonography of the Shoulder: Pitfalls and Variants. Canadian Association of Radiologists Journal, 53(1), pp. 22-32.

32. Jones, A.O. (1998) Magnetic Resonance Imaging of the Supraspinatus Tendon: The Significance of Signal Intensity Alterations at the Critical Zone. Australasian Radiology, 42 (2), pp. 106113.http://dx.doi.org/10.1111/i.1440-1673.1998.tb00584.x

33. Long, C.D. and Nyland, T.G. (1999) Ultrasonographic Evaluation of the Canine Shoulder. Veterinary Radiology \& Ultrasound, 40 (4), pp. 372-379. http://dx.doi.org/10.1111/i.1740-8261.1999.tb02129.x

34. Kramer, M. and Gerwing, M. (1996) The Importance of Sonography in Orthopaedics for Dogs. Berliner und Munchener Tierarztliche Wochenschrift, 109 (4), pp. 130-135.

35. Kramer, M. et al. (2001) Ultrasonography for the Diagnosis of Diseases of the Tendon and Tendon Sheath of the Biceps Brachii Muscle. Veterinary Surgery, 30 (1), pp. 64-

71.http://dx.doi.org/10.1053/ivet.2001.20336

36. Mistieri, M.L. et al. (2012) Ultrasonographic Evaluation of Canine Supraspinatus Calcifying Tendinosis. Journal of the American Animal Hospital Association, 48 (6), pp. 405410.http://dx.doi.org/10.5326/JAAHA-MS-5818

37. Iannotti, J.P. et al. (2005) Accuracy of Office-Based Ultrasonography of the Shoulder for the Diagnosis 
of Rotator Cuff Tears. The Journal of Bone and Joint Surgery (American), 87 (6) pp. 13051311.http://dx.doi.org/10.2106/JBJS.D.02100

38. Ottenheijm, R.P.G. et al. (2014) Ultrasound-Diagnosed Disorders in Shoulder Patients in Daily General Practice: A Retrospective Observational Study. BMC Family Practice, 15 (1) pp.

1115.http://dx.doi.org/10.1186/1471-2296-15-115

39. Smith, T.O. et al. (2011) Diagnostic Accuracy of Ultrasound for Rotator Cuff Tears in Adults: A Systemic Review and Meta-Analysis. Clinical Radiology, 66 (11), pp. 1036-

1048.http://dx.doi.org/10.1016/i.crad.2011.05.007

40. Teefey, S.A. et al. (2004) Detection and Quantification of Rotator Cuff Tears. The Journal of Bone \& Joint Surgery, 86 (4), pp. 708-716.

41. Brose, S.W. et al. (2008) Shoulder Ultrasound Abnormalities, Physical Examination Findings, and Pain in Manual Wheelchair Users with Spinal Cord Injury. Archives of Physical Medicine and Rehabilitation, 89 (11) pp. 2086-2093. http://dx.doi.org/10.1016/j.apmr.2008.05.015

42. Danova, N.A. and Muir, P. (2003) Extracorporeal Shock Wave Therapy for Supraspinatus Calcifying Tendinopathy in Two Dogs. Veterinary Record, 152 (7), pp. 208-

209.http://dx.doi.org/10.1136/vr.152.7.208

43. Benell, K. S. et al. (2007) Efficacy and Cost-Effectiveness of a Physiotherapy Program for Chronic Rotator Cuff Pathology: A Protocol for Randomized, Double-Blind, Placebo-Controlled Trial. BMC Musculoskeletal Disorders, 8 (1), pp. 86. http://dx.doi.org/10.1186/1471-2474-8-86

44. Cookson, J.C. and Kent, B.E. (1979) Orthopaedic Manual Therapy, an Overview. Part 1. The Extremities. Physical Therapy, 59 (2), pp. 136-146.

45. Devor, M. and Sorby, R. (2006) Fibrotic Contracture of the Canine Infraspinatus Muscle: Pathophysiology and Prevention by Early Surgical Intervention. Veterinary and Comparative Orthopaedics and Traumatology, 19, pp. 117-121.

46. Kesikburun, S. et al. (2013) Platelet-Rich Plasma Injections in the Treatment of Chronic Rotator Cuff Tendinopathy: A Randomized Controlled Trial with 1-Year Follow Up. The American Journal of Sports Medicine, 41 (11), pp. 2609-2616 http://dx.doi.org/10.1177/0363546513496542

47. Canapp, S.O. (2013) Regenerative Medicine for Tendinopathies in the Canine. In: Proceedings ACVS Symposium. San Antonio, TX 24-26 April. American College of Veterinary Sports Medicine and Rehabilitation, pp. 343-347.

48. Brosseau, L. et al. (2002) Deep Transverse Friction Massage for Treating Tendonitis. Cochrane Database System Reviews (4):CD003528. http://dx.doi.org/10.1002/14651858.CD003528

49. Wernham, B.G. Warman, G.A. and Jarram, R.M. (2008) Bicipital Tenosynovitis in Dogs. Compendium on Continuing Education for the Practising Veterinarian, 30 (10), pp. 537-552.

50. Gilley, R.S. Wallace, L.J. and Hayden, D.W. (2002) Clinical and Pathologic Analyses of Bicipital Tenosynovitis in Dogs. American Journal of Veterinary Research, 63 (3), pp. 402-

407.http://dx.doi.org/10.2460/ajvr.2002.63.402

51. Jo, C.H. et al. (2011) Does Platelet-Rich Plasma Accelerate Recovery after Rotator Cuff Repair: A Prospective Cohort Study. The American Journal of Sports Medicine, 39 (10), pp. 2082-

2090.http://dx.doi.org/10.1177/0363546511413454 
Table 1. Breed representation of 327 dogs with supraspinatus tendinopathy

\begin{tabular}{|c|c|}
\hline Breed & Number of dogs \\
\hline Labrador Retriever & 70 \\
\hline Mixed Breed & 42 \\
\hline Border Collie & 28 \\
\hline Golden Retriever & 23 \\
\hline German Shepherd Dog & 17 \\
\hline Australian Shepherd & 11 \\
\hline Corgi & 10 \\
\hline Rottweiler & 9 \\
\hline Bernese Mountain Dog & 8 \\
\hline Doberman Pinscher & 8 \\
\hline German Shorthaired Pointer & 7 \\
\hline Standard Poodle & 7 \\
\hline Boxer & 6 \\
\hline Shetland Sheepdog & 6 \\
\hline Greater Swiss Mountain Dog & 5 \\
\hline Chesapeake Bay Retriever & 4 \\
\hline Other* & 66 \\
\hline
\end{tabular}

Figure 1. Shoulder radiograph with mineralisation of the supraspinatus tendon insertion.

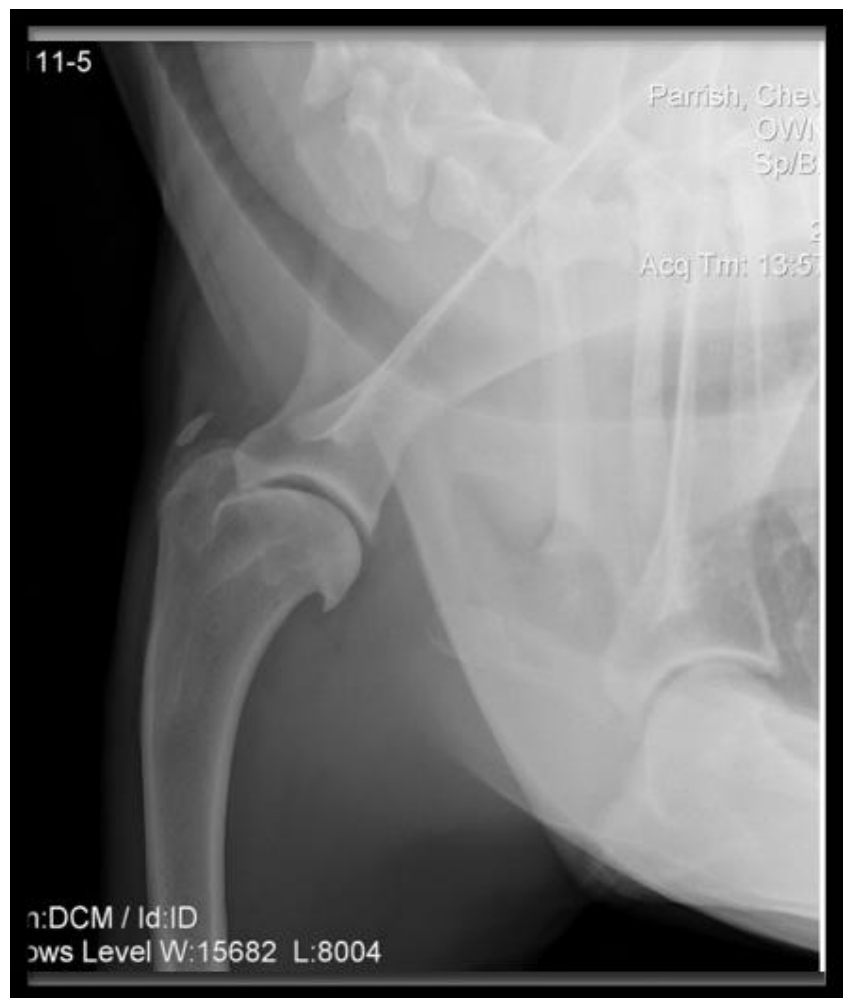


Figure 2. STIR sagittal image of a shoulder MRI in a patient with a ST showing an inflamed supraspinatus tendon.

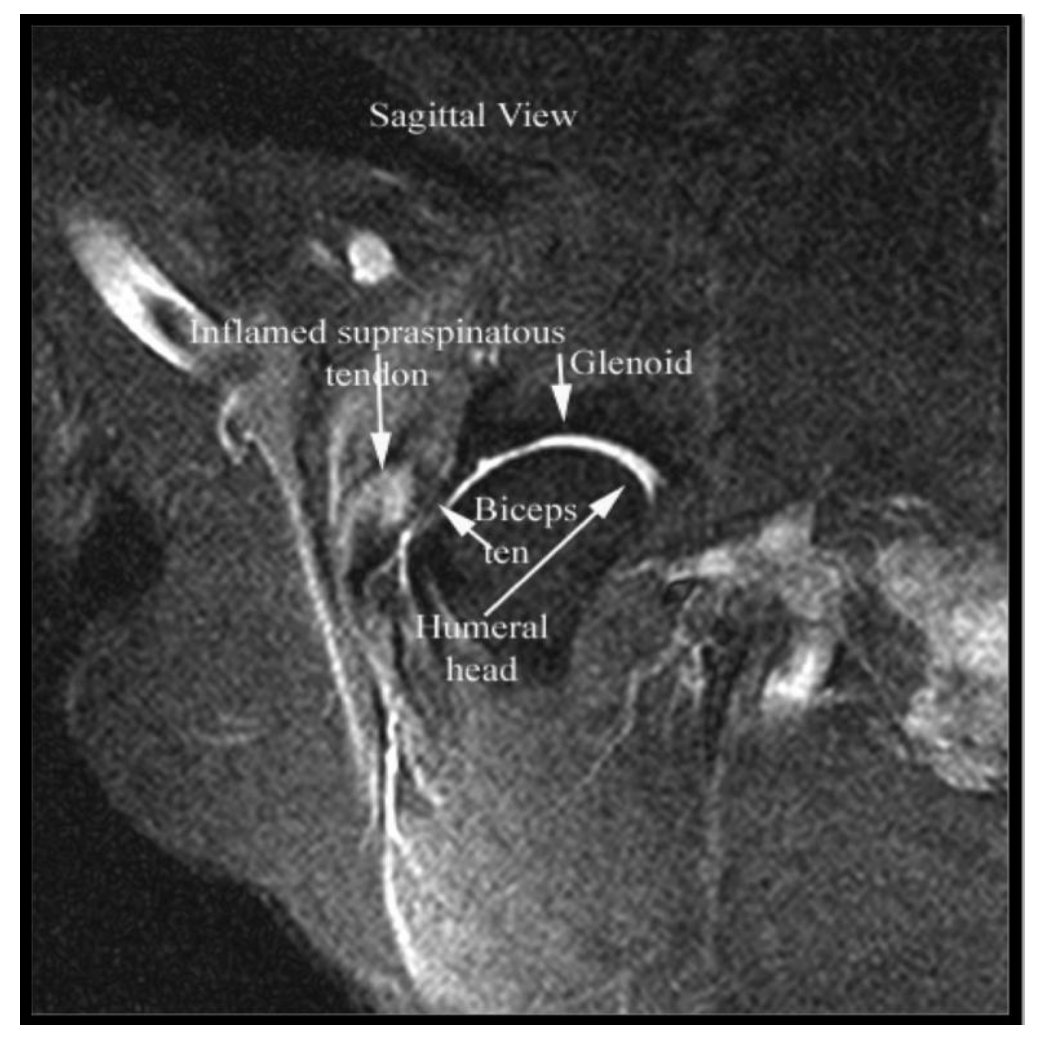

Figure 3. STIR transverse image of a shoulder MRI in a patient with a ST showing a flattened or oval appearance of the biceps tendon and loss of fluid around the biceps tendon within the bicipital groove at the level of insertion of the supraspinatus.

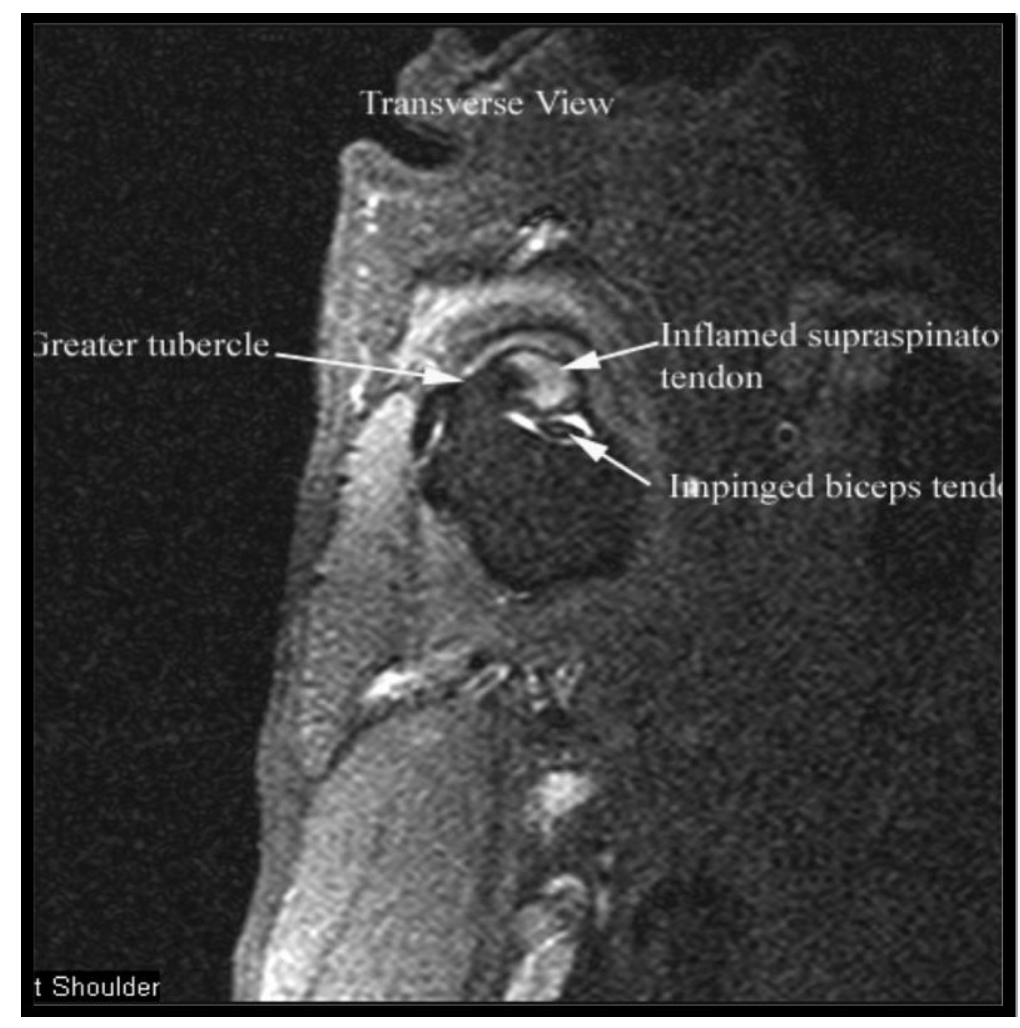


Figure 4. T1 MRI image of a patient with a ST showing fatty replacement at the myotendinous junction of the supraspinatus tendon.

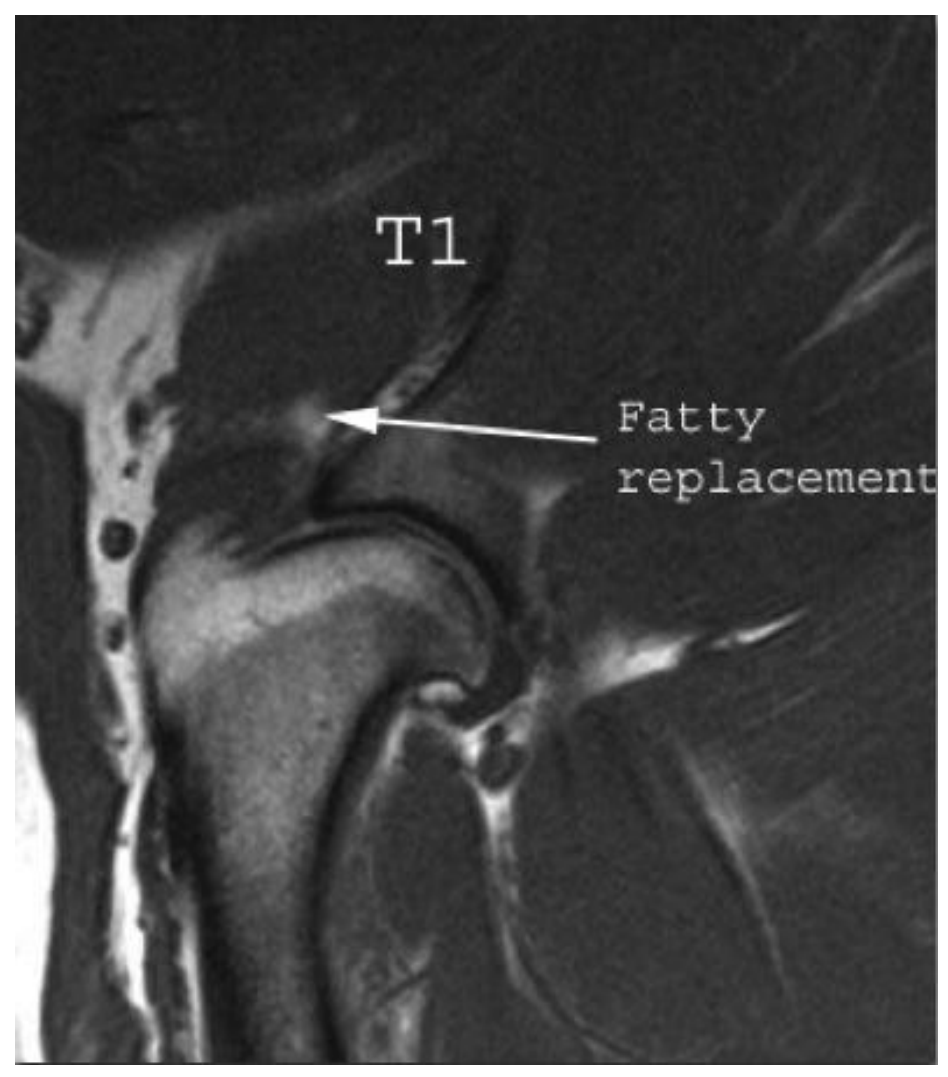

Figure 5. Diagnostic musculoskeletal shoulder ultrasound image depicting an enlargement of the supraspinatus tendon.

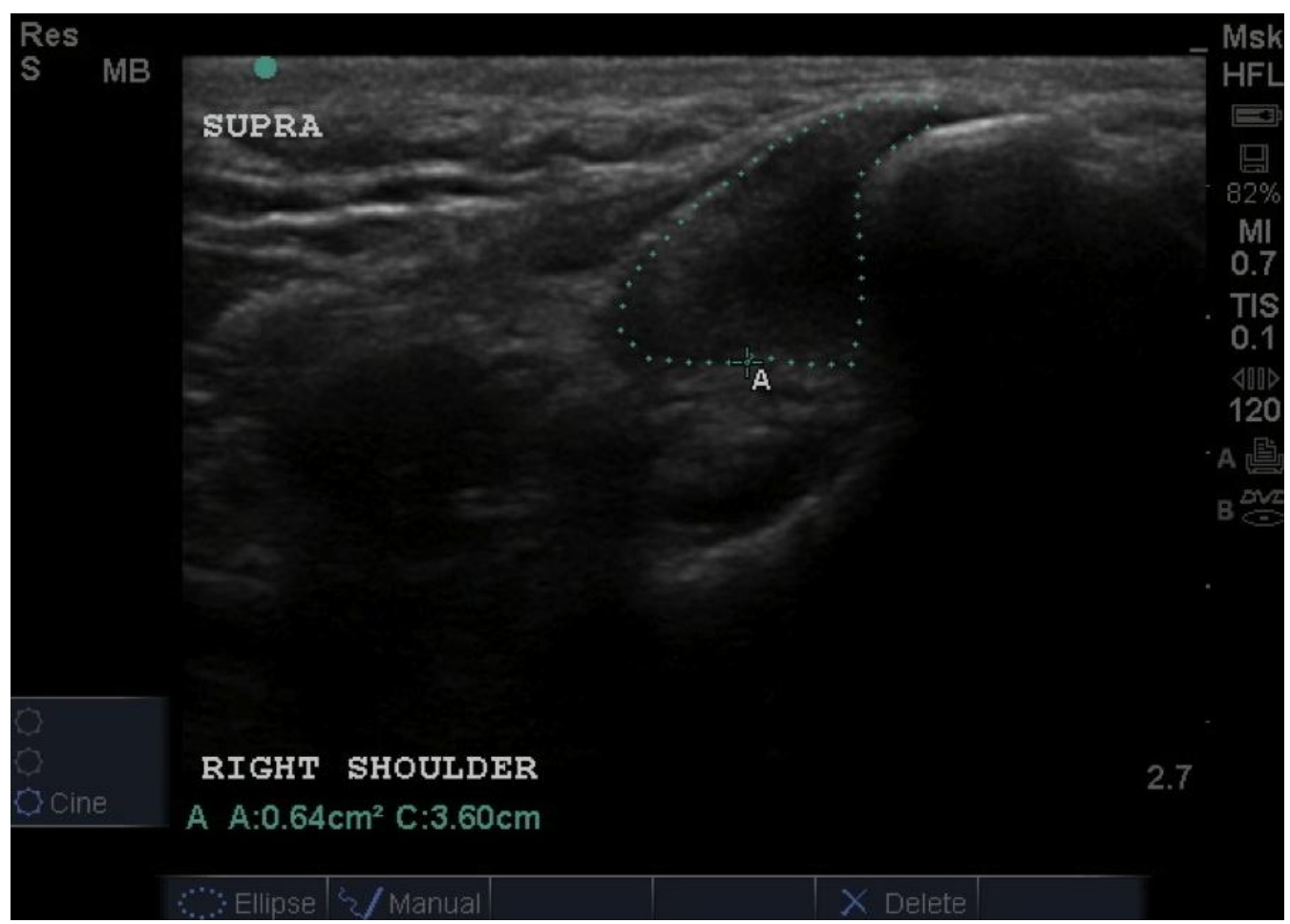


Figure 6. Diagnostic musculoskeletal shoulder ultrasound image showing a supraspinatus tendon with a mixed echogenicity, suggestive of active inflammation.

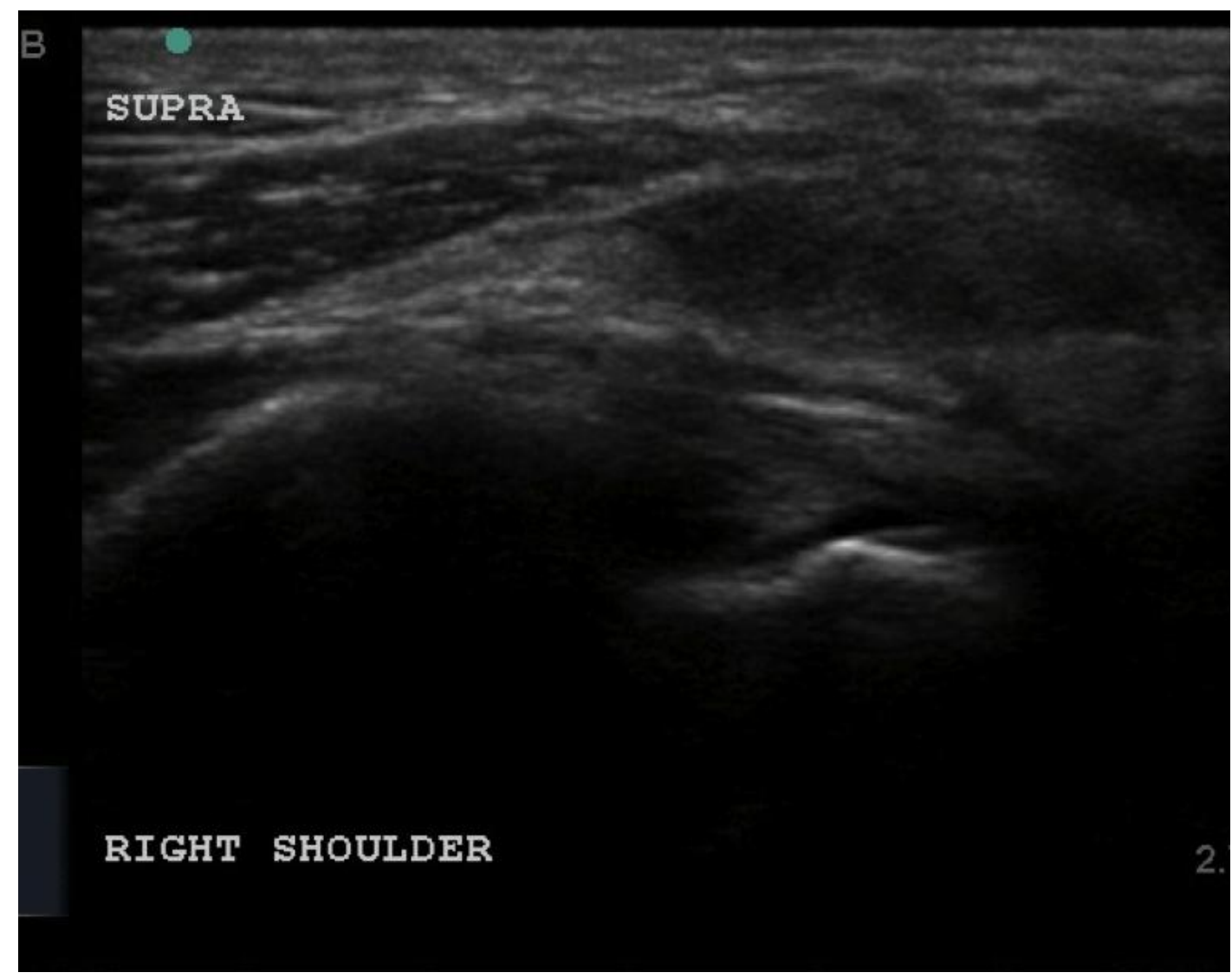

Figure 7. Shoulder arthroscopy demonstrating a supraspinatus bulge and secondary biceps impingement.

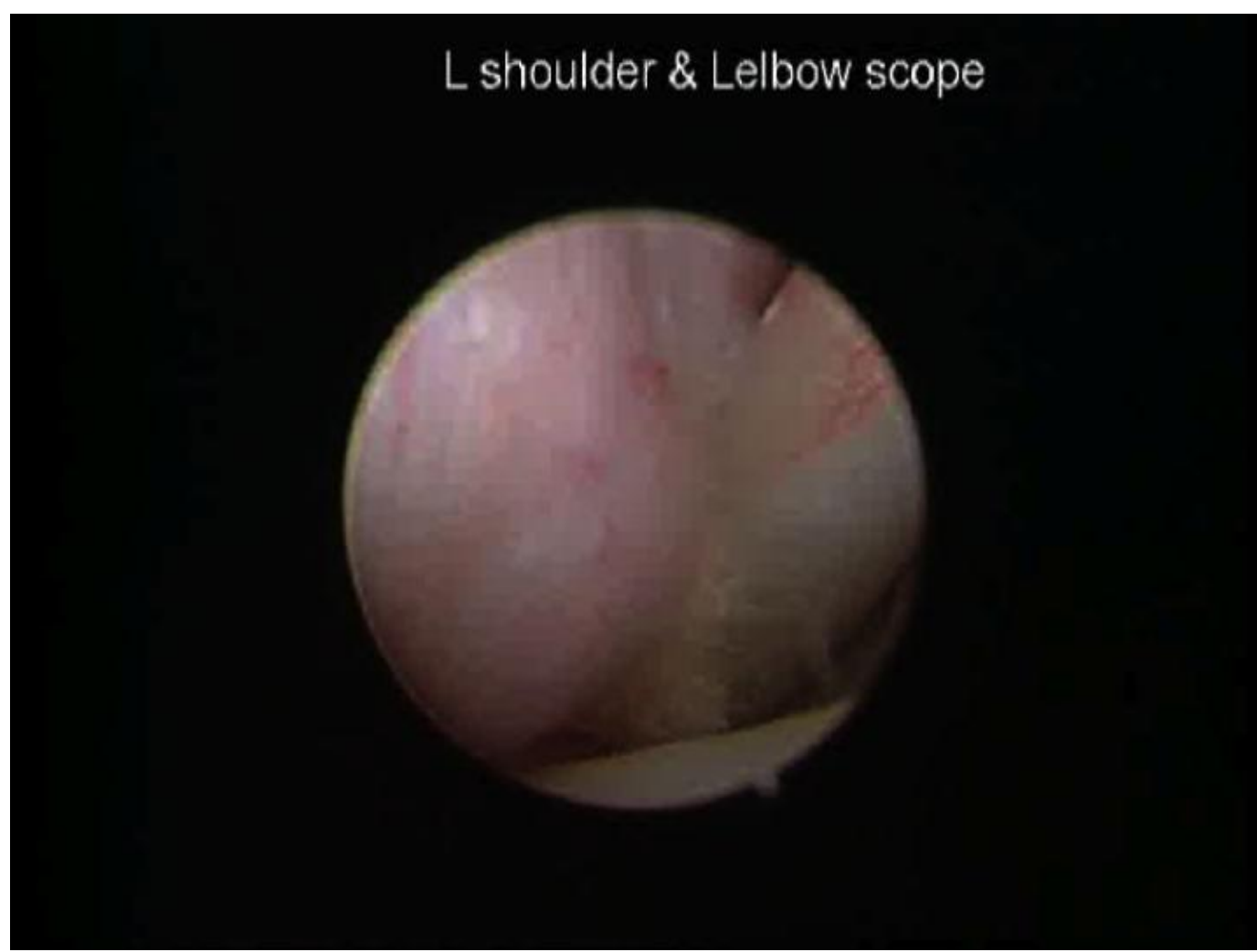




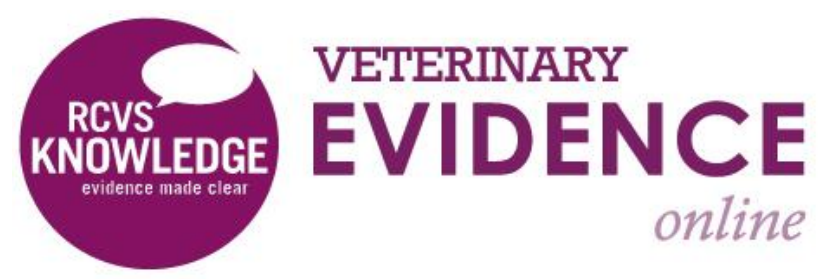

\section{Intellectual Property Rights}

Authors of Articles submitted to RCVS Knowledge for publication will retain copyright in their work, but will be required to grant to RCVS Knowledge an exclusive license of the rights of copyright in the materials including but not limited to the right to publish, re-publish, transmit, sell, distribute and otherwise use the materials in all languages and all media throughout the world, and to license or permit others to do so.

Authors will be required to complete a license for publication form, and will in return retain certain rights as detailed on the form.

Veterinary Evidence and EBVM Network are RCVS Knowledge initiatives. For more information please contact us at editor@veterinaryevidence.org.

RCVS Knowledge is the independent charity associated with the Royal College of Veterinary Surgeons (RCVS). Our ambition is to become a global intermediary for evidence based veterinary knowledge by providing access to information that is of immediate value to practicing veterinary professionals and directly contributes to evidence based clinical decision-making.

www.veterinaryevidence.org

RCVS Knowledge is a registered Charity No. 230886. Registered as a Company limited by guarantee in England and Wales No. 598443.

Registered Office:

Belgravia House

62-64 Horseferry Road

London SW1P 2AF

This work is licensed under a Creative Commons Attribution 4.0 International License. 